ESAIM: M2AN 50 (2016) 77-91

DOI: $10.1051 / \mathrm{m} 2 \mathrm{an} / 2015032$
ESAIM: Mathematical Modelling and Numerical Analysis

www.esaim-m2an.org

\title{
A RELATION BETWEEN A DYNAMIC FRACTURE MODEL AND QUASI-STATIC EVOLUTION
}

\author{
HenRiQue VersieuX ${ }^{1}$
}

\begin{abstract}
We study the relations between a dynamic model proposed by Bourdin, Larsen and Richardson, and quasi-static fracture evolution. We assume the dynamic model has the boundary displacements of the material as input, and consider time-rescaled solutions of this model associated to a sequence of boundary conditions with speed going to zero. Next, we study whether this rescaled sequence converges to a function satisfying quasi-static fracture evolution. Under some hypotheses and assuming the speed of crack propagation slows down following the deceleration of boundary displacements, our main result shows that (up to a subsequence) the rescaled solutions converge to a quasi-static evolution.
\end{abstract}

Mathematics Subject Classification. 35Q74, 74R10, 74R15.

Received May 5, 2014. Revised January 13, 2015.

Published online November 16, 2015.

\section{INTRODUCTION}

The rigorous mathematical formulation of crack propagation models has been an active research area recently. For instance, a considerable effort has been done in order to prove existence of solution for the Francfort and Marigo [10] model for quasi-static crack evolution; see for example [5,7,8,11] and references therein. Due to the mathematical challenges involved, the rigorous formulation of dynamic fracture evolution remains much less developed. To the best of our knowledge the dynamic model proposed by Bourdin et al. [3], was the first model contemplated with an existence of solution result; see [16].

In this work we study relations between the solutions of the Bourdin, Larsen and Richardson dynamic model and quasi-static fracture evolution. More precisely, assume the dynamic model has as input the boundary displacements of the material. Next, consider a sequence of solutions of the dynamic model associated with a sequence of boundary displacements with speed going to zero. We are interested in studying the time-rescaled limit of this sequence of solutions. This problem can be viewed as studying the limit with vanishing viscosity and inertia terms of the Bourdin, Larsen and Richardson dynamical model solutions; see equation (2.11) below. Here it is important to observe that our main result is proved under special assumptions preventing discontinuities in the limit. Also, a similar problem in a different context has been recently addressed in [19].

The starting point of the models considered here is the Francfort and Marigo model for quasi-static fracture evolution, which is based on Griffith's theory [13]. Considering the antiplane case, and assuming $u(t): \Omega \rightarrow \mathbb{R}$

Keywords and phrases. Dynamic fracture model, quasi-static fracture model, energy balance, vanishing viscosity.

1 Instituto de Matemática, Universidade Federal do Rio de Janeiro, Brasil. henrique@im.ufrj.br; versieux@gmx.com 
represents the displacement of a given material, the model states that the crack propagation should be governed by the competition of bulk energy and surface energy as follows. Set

$$
E(u, \Gamma)=\int_{\Omega \backslash \Gamma} \frac{\nu}{2}|\nabla u|^{2} \mathrm{~d} x+\int_{\Gamma} G_{c} \mathrm{~d} \mathcal{H}^{N-1}(\Gamma) .
$$

Here $\mathcal{H}^{N-1}$ is the $N-1$ dimensional Hausdorff measure, $G_{c}$ represents the toughness of the material, $u$ satisfies a boundary condition $\left.u(t)\right|_{\partial \Omega}=g(t)$, and $\Omega$ is an open domain with Lipschitz boundary $\partial \Omega$. The set $\Gamma(t) \subset \Omega$ represents the fracture in this material. Also, the evolution should satisfy: (i) $\Gamma(t)$ is nondecreasing in time. (ii) The energy is absolutely continuous and satisfies a conservation of energy relation. (iii) At time $t$ the crack $\Gamma(t)$ must minimize the total energy $E$ for all displacements $\left.v(t)\right|_{\partial \Omega}=g(t)$ and all fractures $\Gamma$ containing $\cup_{s<t} \Gamma(s)$. A precise mathematical formulation of this model can be found in [11]. The numerical approximation of the Francfort and Marigo model is not straightforward. Rather than a direct numerical approximation of the above minimization problem, a very successful strategy was proposed by Bourdin, Francfort and Marigo, based on the Ambrosio-Tortorelli [1] approximation of the energy functional $E$. This regularization was proved to converge to the solution of the Francfort and Marigo model; see [12]. It is based on the introduction of a new function $v$ and a functional associated to it, such that $v$ captures the discontinuities of the displacement function $u$ and represents the fracture. The dynamic model proposed by Bourdin et al. [3] is based on the Ambrosio-Tortorelli regularization of the Francfort and Marigo model.

The problem we are interested is the following. Consider a smooth function $g:[0,1] \times \Omega \rightarrow \mathbb{R}$, and let $u_{\epsilon}$ and $v_{\epsilon}$ be a solution of the Bourdin, Larsen and Richardson model with Dirichlet boundary condition $g_{\epsilon}(t, x)=g(\epsilon t, x)$. Here $\epsilon>0$ is a parameter controlling the speed of the boundary displacements. Define the time-rescaled functions $u^{\epsilon}(s)=u_{\epsilon}(s / \epsilon)$ and $v^{\epsilon}(s)=v_{\epsilon}(s / \epsilon)$. The functions $g_{\epsilon}, u_{\epsilon}$, and $v_{\epsilon}$ are defined in $[0,1 / \epsilon]$ while $g^{\epsilon}, u^{\epsilon}$, and $v^{\epsilon}$ are defined in $[0,1]$, also $\left.u^{\epsilon}(s)\right|_{\partial \Omega}=\left.g(s)\right|_{\partial \Omega}, s \in[0,1]$. Our main interest is to understand the limits of $u^{\epsilon}$ and $v^{\epsilon}$ when $\epsilon$ goes to zero. One could expect that such functions have limits satisfying a quasi-static model. Nevertheless, it is not straightforward to see which is the precise quasi-static model satisfied in the limit. Since the Bourdin, Larsen and Richardson dynamic model is based on the regularized Francfort and Marigo model, one could think on the later as a candidate for the quasi-static model satisfied by the limit of the rescaled functions. As a matter of fact, we show under some assumptions that the limits of $u^{*}$ of $u^{\epsilon}$ and $v^{*}$ of $v^{\epsilon}$ satisfy, irreversibility condition and the same conservation of energy from the regularized Francfort and Marigo model. However, here we obtain that the limits $u^{*}$ and $v^{*}$ separately minimize the energy, rather than been a global minimizer as in the Francfort and Marigo model (see Sect. 2.4).

Our first step is to obtain bounds for the families $u^{\epsilon}$, and $v^{\epsilon}$ such that a compactness result can be applied to extract a converging sequence. This is done by revisiting the a priori estimates from [16], and understanding the dependence of these estimates on $\epsilon$. We are able to obtain bounds independent of $\epsilon$ for $u^{\epsilon}$ and $v^{\epsilon}$ in $L^{\infty}\left(0,1 ; L^{2}(\Omega)\right)$ and $L^{\infty}\left(0,1 ; H^{1}(\Omega)\right)$, respectively (see Lem. 3.3).

Next, we study when the functions obtained as weak limits of $u^{\epsilon}$, and $v^{\epsilon}$ in the previous step satisfy the conservation of energy condition from the Ambrosio-Tortorelli regularization of the Francfort and Marigo model. This step represents the core of our work. Under some technical conditions, and assuming that the maximum speed of crack propagation for the dynamic model slows down following the deceleration of boundary displacements (more precisely, $\partial_{s} v^{\epsilon} \in L^{2}\left(0,1 ; L^{\infty}(\Omega)\right)$ ) we show that the obtained limits satisfy the conservation of energy (see Thm. 4.7).

Finally, we obtain conditions implying the limits $u^{*}$ and $v^{*}$ from the first step separately minimize the energy functional. Together these three steps yield our main result (see Thm. 6.1).

This paper is organized as follows. Section 2 presents the regularized quasi-static model, the dynamic model, and the rescaled solutions of the dynamic model. It also introduces an alternative quasi-static model satisfied by the limit of our rescaled in time solutions. Section 3 provides bounds for the sequence of rescaled solutions. In Section 4, we study when the limit of the rescaled solutions satisfies the conservation of energy condition of the quasi-static model. Section 5 presents conditions such the rescaled solutions converge to functions satisfying a minimality condition. Finally, Section 6 states our main result. 
Notation. We use $\langle\cdot, \cdot\rangle$ to denote the $L^{2}(\Omega)$ (or $L^{2}(\Omega)^{n}$ ) inner product or the pairing between $H_{0}^{1}(\Omega)$ and $H^{-1}(\Omega)$. We denote the $L^{2}(\Omega)$ norm by $\|\cdot\|$, and the $W^{k, p}(\Omega)$ norm (seminorm) by $\|\cdot\|_{k, p}\left(|\cdot|_{k, p}\right)$. Also, in the case $p=2$ we use the notation $\|\cdot\|_{k}\left(|\cdot|_{k}\right)$ for the $H^{k}(\Omega)$ norms (seminorms). The spaces $L^{p}\left(0, T ; H^{1}(\Omega)\right)$ are denoted $L^{p}\left(0, T ; H^{1}\right)$, similarly in other cases involving space and time spaces. The subindex $\epsilon$ represents a sequence (or a subsequence) $\left\{\epsilon_{j}\right\}$ converging to zero. We denote a generic constant independent of $\epsilon$ by $c$. There are two time variables $t$ and $s$, respecting the relation $s=\epsilon t$. We use the notation $\dot{u}$ only for the time derivative of $u$ with respect to $s\left(\dot{u}=\partial_{s} u\right)$.

\section{Crack propagation models AND Rescaled SOlutions}

In this section, we introduce the models that are employed in our work. We consider the antiplane case, and assume the displacement of a given material is represented by a function $u(t): \Omega \rightarrow \mathbb{R}$, satisfying a prescribed boundary condition $\left.u(t)\right|_{\partial \Omega}=g(t)$. Here $\Omega \subset \mathbb{R}^{2}$ is a bounded open set with Lipschitz continuous boundary. First, we introduce the regularized Francfort and Marigo quasi-static model. Next, we present the regularized dynamic model, and subsequently define the rescaled in time solutions of this model. Finally, we define the quasi-static model satisfied by the limit of the sequence of rescaled solutions.

\subsection{The regularized quasi-static model}

The regularized quasi-static evolution considered here was introduced by Bourdin et al. [2]. It is based on the Ambrosio-Tortorelli [1] approximation of the quasi-static model proposed by Francfort and Marigo [10]. In order to introduce the model, we first define the following functionals

$$
\begin{aligned}
\mathcal{E}(u, v) & =\frac{1}{2} \int_{\Omega}\left(v^{2}+\eta_{\gamma}\right)|\nabla u|^{2} \mathrm{~d} x \text { and } \mathcal{H}(v)=\int_{\Omega} \frac{1}{4 \gamma}(1-v)^{2}+\gamma|\nabla v|^{2} \mathrm{~d} x \\
E_{\gamma}(u, v) & =\mathcal{E}(u, v)+\mathcal{H}(v) .
\end{aligned}
$$

Here $v$ is a function satisfying $0 \leq v \leq 1$, and $0<\eta_{\gamma} \ll \gamma$ are the parameters of the Ambrosio-Tortorelli approximation of the functional $E(u)$ defined in (1.1), in the sense that $E_{\gamma} \Gamma$-converges to $E$ when $\gamma \rightarrow 0$; see $[4,9,12]$. Also, in the limit $\gamma \rightarrow 0$ the set $\{x \in \Omega ; v(x)=0\}$ approximates the set $\Gamma$ (the points of discontinuities of $u$ ), and represents the fracture. The model is given by functions $s \mapsto u(s), v(s)$ such that for every $s \in[0,1]$

(a): $u(s)=g(s)$ on $\partial \Omega, 0 \leq v \leq 1$

(b): for all $0 \leq s^{\prime} \leq s \leq 1 ; v(s) \leq v\left(s^{\prime}\right)$;

(c): for all $(\tilde{u}, \tilde{v}) \in H^{1}(\Omega) \times H^{1}(\Omega)$ with $\tilde{u}=g(s)$ and $\tilde{v}=1$ on $\partial \Omega$, and $0 \leq \tilde{v} \leq v(s)$ we have

$$
\mathcal{E}(u(s), v(s))+\mathcal{H}(v(s)) \leq \mathcal{E}(\tilde{u}, \tilde{v})+\mathcal{H}(\tilde{v})
$$

(d): the function $\mathcal{E}(u(s), v(s))+\mathcal{H}(v(s))$ is absolutely continuous for all $s \in[0,1]$, and

$$
\begin{aligned}
\mathcal{E}(u(s), v(s))+\mathcal{H}(v(s))= & \mathcal{E}(u(0), v(0))+\mathcal{H}(v(0)) \\
& +\int_{0}^{s}\left\langle\left(\eta_{\gamma}+v^{2}\right) \nabla u, \nabla g_{\tau}\right\rangle \mathrm{d} \tau
\end{aligned}
$$

(e): there exists a constant $c>0$ such that $\mathcal{E}(u(s), v(s))+\mathcal{H}(v(s)) \leq c$ for all $s \in[0,1]$.

\subsection{The dynamic model}

Here we employ a version of the model studied by Larsen et al. [16] simplified to our purposes. In order to introduce the model, along with the functionals $\mathcal{E}$ and $\mathcal{H}$ defined by (2.1) we also introduce the kinetic energy

$$
\mathcal{K}\left(u_{t}\right)=\frac{1}{2} \int_{\Omega}\left|u_{t}\right|^{2} \mathrm{~d} x
$$


and the total energy

$$
\mathcal{F}\left(u, v, u_{t}\right)=\mathcal{E}(u, v)+\mathcal{H}(v)+\mathcal{K}\left(u_{t}\right) .
$$

Given a time $T_{f}>0$ the model is given by a pair of functions $u$ and $v$ satisfying the relations:

$$
\begin{aligned}
u_{t t}-\nabla \cdot\left(a(t) \nabla\left(u+u_{t}\right)\right) & =0 \text { in } \Omega \\
u(t) & =g(t) \text { on } \partial \Omega \\
u(0) & =\rho_{0}, \quad u_{t}(0)=\rho_{1} \text { and } v(0)=v_{0}
\end{aligned}
$$

for $t \in\left[0, T_{f}\right]$. Where $g \in H^{2}\left(0, T_{f} ; H^{1}\right)$,

$$
a(t)=v(t)^{2}+\eta_{\gamma}
$$

$\rho_{0}, \rho_{1}$, and $v_{0} \in H^{1}(\Omega), 0 \leq \mu v_{0} \leq 1$ are known. Also, $u$ and $v$ satisfy

$$
\mathcal{E}(u(t), v(t))+\mathcal{H}(v(t))=\inf _{v \leq v(t)} \mathcal{E}(u(t), v)+\mathcal{H}(v)
$$

and the conservation of energy

$$
\begin{aligned}
\mathcal{F}\left(u(T), v(T), u_{t}(T)\right)= & \mathcal{F}\left(u(0), v(0), u_{t}(0)\right)-\int_{0}^{T}\left\|a^{1 / 2} \nabla u_{t}\right\|^{2} \\
& +\int_{0}^{T}\left\langle u_{t t}, g_{t}\right\rangle+\left\langle a \nabla\left(u+u_{t}\right), \nabla g_{t}\right\rangle \mathrm{d} t
\end{aligned}
$$

for $T \in\left[0, T_{f}\right]$.

The following theorem from [16] guarantees existence of a solution for the dynamic model.

Theorem 2.1. Let $T_{f}>0$ and assume $g \in H^{2}\left(0, T_{f} ; H^{1}\right)$, then there exists at least one curve $(u, v) \in$ $\left(H^{2}\left(0, T_{f} ; L^{2}\right) \cap W^{1, \infty}\left(1, T_{f} ; H^{1}\right)\right) \times W^{1, \infty}\left(0, T_{f} ; H^{1}\right)$ such that

$$
\left\langle u_{t t}, \phi\right\rangle+\left\langle a \nabla\left(u+u_{t}\right), \nabla \phi\right\rangle=0 \quad \forall \phi \in H_{0}^{1}(\Omega) \quad \text { a.e. } t \in\left(0, T_{f}\right)
$$

$u(t)-g(t) \in H_{0}^{1}(\Omega), u(0)=\rho_{0}, u_{t}=\rho_{1}, v(0)=v_{0}$, and conditions (2.6), and (2.7) are satisfied.

Proof. See Theorem 2.1 and Remarks 2.1 and 2.2 from [16].

\subsection{Rescaled solutions of the dynamic problem}

Consider a sequence of boundary conditions for the dynamic problem defined by

$$
g_{\epsilon}(t)=g(t \epsilon), \quad \text { where } g \in H^{2}\left(0,1 ; H^{1}\right) \text { and } \epsilon>0 .
$$

Hence, the speed of boundary displacements goes to zero as $\epsilon$ goes to zero.

Definition 2.2. Set the functions $\left(u_{\epsilon}, v_{\epsilon}\right):[0,1 / \epsilon] \rightarrow H^{1}(\Omega) \times H^{1}(\Omega)$ as a solution for problem (2.4), given by Theorem 2.1, with boundary condition $\left.u_{\epsilon}(t)\right|_{\partial \Omega}=g_{\epsilon}(t)$ and initial condition $u_{\epsilon}(0)=\rho_{0}, \partial_{t} u_{\epsilon}(0)=\epsilon \rho_{1}$ and $v_{\epsilon}(0)=v_{0}$.

Also, we introduce the rescaled functions associated to $\left(u_{\epsilon}, v_{\epsilon}\right)$ :

Definition 2.3. Set $\left(u^{\epsilon}, v^{\epsilon}\right):[0,1] \rightarrow H^{1}(\Omega) \times H^{1}(\Omega)$ as:

$$
u^{\epsilon}(s)=u_{\epsilon}(s / \epsilon) \text { and } v^{\epsilon}(s)=v_{\epsilon}(s / \epsilon)
$$

We also set $a^{\epsilon}(s)=v^{\epsilon}(s)^{2}+\eta_{\gamma}$. In order to simplify our notation we denote $\dot{u}^{\epsilon}=\partial_{s} u^{\epsilon}(s)$ and $\ddot{u}^{\epsilon}=\partial_{s}^{2} u^{\epsilon}(s)$ (similarly for other functions depending on $s$ ). 
The rescaled sequence of functions $u^{\epsilon}(s)$ satisfies the boundary condition $\left.u^{\epsilon}(s)\right|_{\partial \Omega}=\left.g(s)\right|_{\partial \Omega}$ for $s \in[0,1]$. Our main goal is to obtain conditions such that the sequence $\left(u^{\epsilon}, v^{\epsilon}\right)$ has a limit satisfying the regularized quasi-static model associated with boundary conditions $g$.

A few properties of the functions $u^{\epsilon}$ and $v^{\epsilon}$ are now derived. Using that $s=\epsilon t$ we have

$$
u^{\epsilon}(\epsilon t)=u_{\epsilon}(t) \text { for } t \in[0,1 / \epsilon]
$$

and hence

$$
\epsilon \partial_{s} u^{\epsilon}(\epsilon t)=\partial_{t} u_{\epsilon}(t) \text { and } \epsilon^{2} \partial_{s}^{2} u^{\epsilon}(\epsilon t)=\partial_{t}^{2} u_{\epsilon}(t) .
$$

Since $u_{\epsilon}$ and $v_{\epsilon}$ satisfy the weak formulation for the dynamic problem (see $(2.8)$ ) we have for a.e. $s \in[0,1]$

$$
\epsilon^{2}\left\langle\ddot{u}^{\epsilon}(s), \varphi\right\rangle+\left\langle a^{\epsilon}(s) \nabla\left(u^{\epsilon}(s)+\epsilon \dot{u}^{\epsilon}(s)\right), \nabla \varphi\right\rangle=0 \quad \forall \varphi \in H_{0}^{1}(\Omega)
$$

where $a^{\epsilon}(s)=a_{\epsilon}(s / \epsilon)$, and we have used the simpler notation $\dot{u}^{\epsilon}=\partial_{s} u^{\epsilon}$ and $\ddot{u}^{\epsilon}=\partial_{s}^{2} u^{\epsilon}$. From (2.6) we obtain

$$
\mathcal{E}\left(u^{\epsilon}(s), v^{\epsilon}(s)\right)+\mathcal{H}\left(v^{\epsilon}(s)\right)=\inf _{v \leq v^{\epsilon}(s)} \mathcal{E}\left(u^{\epsilon}(s), v\right)+\mathcal{H}(v) .
$$

Also, the conservation of energy (2.7) yields

$$
\begin{aligned}
\mathcal{E}\left(u^{\epsilon}(S), v^{\epsilon}(S)\right)+\mathcal{H}\left(v^{\epsilon}(S)\right) & +\epsilon^{2} \mathcal{K}\left(\dot{u}^{\epsilon}(S)\right)=\mathcal{E}\left(u^{\epsilon}(0), v^{\epsilon}(0)\right)+\mathcal{H}\left(v^{\epsilon}(0)\right) \\
+ & +\epsilon^{2} \mathcal{K}\left(\dot{u}^{\epsilon}(0)\right)-\int_{0}^{S} \epsilon\left\|\sqrt{a^{\epsilon}} \nabla \dot{u}^{\epsilon}\right\|^{2} \mathrm{~d} s+\int_{0}^{S} \epsilon^{2}\left(\ddot{u}^{\epsilon}, \dot{g}\right)+\left\langle a^{\epsilon} \nabla\left(u^{\epsilon}+\epsilon \dot{u}^{\epsilon}\right), \nabla \dot{g}\right\rangle \mathrm{d} s
\end{aligned}
$$

for $S \in[0,1]$.

\subsection{The limit regularized quasi-static model}

We now introduce the quasi-static model satisfied by the limit of the rescaled in time functions introduced in the last subsection.

Find $(\tilde{u}, \tilde{v}):[0,1] \rightarrow H^{1}(\Omega) \times H^{1}(\Omega)$ satisfying:

$(\tilde{a}): \tilde{u}(s)=g(s)$ on $\partial \Omega, 0 \leq \tilde{v} \leq 1$;

(b): for all $0 \leq s^{\prime} \leq s \leq 1 ; \tilde{v}(s) \leq \tilde{v}\left(s^{\prime}\right)$;

$(\tilde{\mathrm{c}})$ : we have for $s \in[0,1]$

$$
\mathcal{E}(\tilde{u}(s), \tilde{v}(s))+\mathcal{H}(\tilde{v}(s))=\inf _{0 \leq z \leq \tilde{v}(s)} \mathcal{E}(\tilde{u}(s), z)+\mathcal{H}(z),
$$

and

$$
\mathcal{E}(\tilde{u}(s), \tilde{v}(s))+\mathcal{H}(\tilde{v}(s))=\inf _{\phi-g(s) \in H_{0}^{1}(\Omega)} \mathcal{E}(\phi, \tilde{v}(s))+\mathcal{H}(\tilde{v}(s)) .
$$

$(\tilde{\mathrm{d}})$ : the function $\mathcal{E}(\tilde{u}(s), \tilde{v}(s))+\mathcal{H}(\tilde{v}(s))$ is absolutely continuous for $s \in[0,1]$, and

$$
\mathcal{E}(\tilde{u}(s), \tilde{v}(s))+\mathcal{H}(\tilde{v}(s))=\mathcal{E}(\tilde{u}(0), \tilde{v}(0))+\mathcal{H}(\tilde{v}(0))+\int_{0}^{s}\left\langle\left(\eta_{\gamma}+\tilde{v}^{2}\right) \nabla \tilde{u}, \nabla g_{\tau}\right\rangle \mathrm{d} \tau
$$

$(\tilde{\mathrm{e}}):$ there exists a constant $c>0$ such that $\mathcal{E}(\tilde{u}(s), \tilde{v}(s))+\mathcal{H}(\tilde{v}(s)) \leq c$ for $s \in[0,1]$.

We observe that the minimality condition here is different than the condition in item (c) Section 2.1. The quasi-static model proposed by Francfort and Marigo was based on Griffith's theory, which is local. As pointed out by Francfort and Marigo, the stationary property of Griffith's theory was replaced by a global minimality condition due to the mathematical challenges of treating the former. The separate minimality condition $(\tilde{\mathrm{c}})$ implies that for each $s$ a solution of the limit model $(\tilde{u}(s), \tilde{v}(s))$ is a equilibrium point of the energy, i.e. 
$\partial_{\tilde{v}}(\mathcal{E}(\tilde{u}(s), \tilde{v}(s))+\mathcal{H}(\tilde{v}(s))) \geq 0$ and $\partial_{\tilde{u}} \mathcal{E}(\tilde{u}(s), \tilde{v}(s))=0$, where $\partial_{\tilde{v}}$ denotes the subdifferential operator with respect to $\tilde{v}$ (similarly for $\partial_{\tilde{u}}$ ). Furthermore, the energy is separately quadratic and hence equilibrium points of

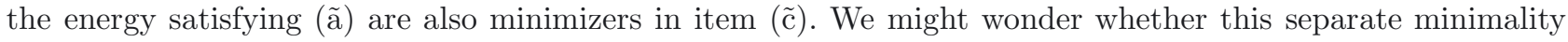
agrees with the global minimality from Section 2.1. This question however, raises issues that are beyond the scope of this work.

A comparison between crack evolution governed by global minimality versus equilibrium point condition (Griffith's criterion) has shown that these two evolutions may not agree; see $[14,18]$. The differences from global minimality versus equilibrium point condition have also been studied in a more general framework of a rate-independent system; see for instance [17] and references therein. More precisely, different concepts of solutions for a rate-independent system are studied in [17]. The Francfort and Marigo model corresponds to an energetic solution (see p. 89 and Def. 3.1 from [17]), whether our separate minimality condition corresponds to the equilibrium point condition of BV solutions (see [17], Def. 4.21). Furthermore, if a BV solution of a rate-independent system has no jumps with respect to the time variable $t$ then it satisfies the same conservation of energy as an energetic solution. We observe that the conservation of energy satisfied by our limit model (item $(\tilde{\mathrm{d}})$ above) is equal to the one satisfied by the regularized approximation of the Francfort and Marigo model (item (d) from Sect. 2.1). Also, under the hypotheses considered here the limit of our rescaled solutions are proven to be continuous with respect to $t$; see the proof of Lemma 4.6 below. These facts suggest that the limit of our rescaled solutions represents a regularized approximation of a BV solution of the rate-independent system associated with the Francfort and Marigo model. Also, as pointed out in [17], given a rate-independent system it is expected that an energetic solution will jump as soon as possible, while a BV solution will jump as late as possible. Finally, other authors have also proposed alternative models for quasi-static fracture propagation based on local minimizers; see for instance $[6,15]$.

\section{A priori estimates}

Here we obtain a bound independent of $\epsilon$ for $u^{\epsilon}$ and $v^{\epsilon}$ introduced in Definition 2.3, yielding the existence of a convergent subsequence through a compactness result. The proof of Theorem 2.1 found in [16] is based on a priori estimates for a discrete-in-time approximation of the model (2.4)-(2.7). In our analysis we apply this theorem to define the functions $u_{\epsilon}$ and $v_{\epsilon}$ on the time interval $\left[0, T_{f}=1 / \epsilon\right]$. For this reason, we next study how some of the a priori estimates from [16] depend on $T_{f}$ and on the boundary condition $g$.

The discrete-in-time approximation for problem (2.4)-(2.7) proposed in [16] is the following. Let $N_{f} \in \mathbb{N}$, and set $v^{0}=v_{0}, a^{0}=\left(v^{0}\right)^{2}+\eta_{\gamma}, u^{0}=\rho_{0}, u^{0}-u^{-1}=h \rho_{1}$, and $g^{n}=g(n h)$ with $h=T_{f} / N_{f}$. For $n=1,2, \ldots, N_{f}$, we recursively define the functions $u^{n}$ and $v^{n}$ as the solutions of the following problem

$$
\begin{aligned}
& \left\langle\delta_{h}^{2} u^{n}, \phi\right\rangle+\left\langle a^{n-1} \nabla\left(u^{n}+\delta_{h} u^{n}\right), \nabla \phi\right\rangle=0, \quad \forall \phi \in H_{0}^{1}(\Omega), \\
& v^{n}=\operatorname{argmin}_{v \leq v^{n-1}}\left\{v \rightarrow \mathcal{E}\left(u^{n}, v\right)+\mathcal{H}(v)\right\}
\end{aligned}
$$

where $a^{n-1}=\left(v^{n-1}\right)^{2}+\eta_{\gamma}, u^{n}-g^{n} \in H_{0}^{1}(\Omega)$ and

$$
\delta_{h} \phi^{n}=\frac{\phi^{n}-\phi^{n-1}}{h} \text { and } \delta_{h}^{2} \phi^{n}=\frac{\phi^{n}-2 \phi^{n-1}+\phi^{n-2}}{h} .
$$

In our analysis we will need the following result from Ortner, Larsen and Sulli (see Lem. 3.2 from [16])

Lemma 3.1. For all $n=1, \ldots, N_{f}$ we have that

$$
\left\langle\left|\nabla u^{n}\right|^{2} v^{n}, \delta_{h} v^{n}\right\rangle+(2 \epsilon)^{-1}\left\langle v^{n}-1, \delta_{h} v^{n}\right\rangle+2 \epsilon\left\langle\nabla v^{n}, \nabla \delta_{h} v^{n}\right\rangle=0 .
$$

The next lemma corresponds to Lemma 3.3 from [16], and presents how the constants on the a priori estimate for the functions $v^{n}$ and $u^{n}$ depend on $T_{f}$. This estimate is important to obtain bounds independent of $\epsilon$ for the rescaled solutions $u^{\epsilon}$ and $v^{\epsilon}$. The proof presented here is adapted to our simplified setting (also it treats the Dirichlet boundary condition case). 
Lemma 3.2. Let $u^{n}$ and $v^{n}$ be defined by (3.1) and (3.2), then the following estimate holds for all $1 \leq N \leq N_{f}$.

$$
\begin{aligned}
\max _{1 \leq n \leq N}\left\|\delta_{h} u^{n}\right\|^{2}+\max _{1 \leq n \leq N}\left\|\nabla u^{n}\right\|^{2}+\max _{1 \leq n \leq N}\left\|v^{n}\right\|^{2} & +\max _{1 \leq n \leq N}\left\|\nabla v^{n}\right\|^{2} \\
& +\sum_{n=1}^{N}\left\|\left(a^{n-1}\right)^{1 / 2} \nabla \delta_{h} u^{n}\right\|^{2}+h \sum_{n=1}^{N} h D_{h}^{n} \leq I\left(g, \rho_{0}, v_{0}, \rho_{1}\right)
\end{aligned}
$$

where

$$
\begin{aligned}
I\left(g, \rho_{0}, v_{0}, \rho_{1}\right)= & \mathcal{F}\left(\rho_{0}, v_{0}, \rho_{1}\right)+\left\|\delta_{h} u^{0}\right\|\left\|\delta_{h} g^{1}\right\|+c_{T_{f}} \sum_{n=1}^{N} h\left\|\delta_{h}^{2} g^{n+1}\right\|^{2} \\
& +\left(c+c_{T_{f}}\right) \sum_{n=1}^{N} h\left\|\nabla \delta_{h} g^{n}\right\|^{2}
\end{aligned}
$$

and $D_{n}^{h} \geq 0$; see (3.12) below. Furthermore, the constant $c_{T_{f}}$ grows linearly with $T_{f}$ :

$$
c_{T_{f}} \leq c+c T_{f} .
$$

Proof. Choose $\phi=h \delta_{h}\left(u^{n}-g^{n}\right)$ in (3.1) to obtain:

$$
\left\langle\delta_{h} u^{n}-\delta_{h} u^{n-1}, \delta_{h}\left(u^{n}-g^{n}\right)\right\rangle+\left\langle a^{n-1} \nabla\left(u^{n}+\delta_{h} u^{n}\right), \nabla h \delta_{h}\left(u^{n}-g^{n}\right)\right\rangle=0
$$

Next, we observe that

$$
\begin{aligned}
\left\langle\delta_{h} u^{n}-\delta_{h} u^{n-1}, \delta_{h} u^{n}\right\rangle= & \frac{1}{2}\left\|\delta_{h} u^{n}\right\|^{2}+\frac{1}{2}\left\|\delta_{h} u^{n}\right\|^{2}-\left\langle\delta_{h} u^{n}, \delta_{h} u^{n-1}\right\rangle \\
& +\frac{1}{2}\left\|\delta_{h} u^{n-1}\right\|^{2}-\frac{1}{2}\left\|\delta_{h} u^{n-1}\right\|^{2}=\mathcal{K}\left(\delta_{h} u^{n}\right)-\mathcal{K}\left(\delta_{h} u^{n-1}\right)+\frac{1}{2} h^{2}\left\|\delta_{h}^{2} u^{n}\right\|^{2}
\end{aligned}
$$

and similarly we have

$$
\begin{aligned}
\left\langle a^{n-1} \nabla u^{n}, \nabla\left(u^{n}-u^{n-1}\right)\right\rangle= & \mathcal{E}\left(u^{n}, v^{n}\right)-\mathcal{E}\left(u^{n-1}, v^{n-1}\right) \\
& +\frac{1}{2} h^{2}\left\|\left.\left(a^{n-1}\right)^{1 / 2} \nabla \delta_{h} u^{n}\left|\|^{2}-\frac{1}{2} \int_{\Omega}\left(a^{n}-a^{n-1}\right)\right| \nabla u^{n}\right|^{2} \mathrm{~d} x .\right.
\end{aligned}
$$

We rewrite the forth term on the right-hand side of the last equation by observing that

$$
a^{n}-a^{n-1}=\left(v^{n}\right)^{2}-\left(v^{n-1}\right)^{2}=h\left(v^{n}+v^{n-1}\right) \delta_{h} v^{n}=2 h v^{n} \delta_{h} v^{n}-h^{2}\left|\delta_{h} v^{n}\right|^{2}
$$

and using Lemma 3.1 to obtain

$$
\begin{aligned}
-\frac{1}{2} \int_{\Omega}\left(a^{n}-a^{n-1}\right)\left|\nabla u^{n}\right|^{2} \mathrm{~d} x= & \frac{1}{2} h^{2}\left\|\left(\delta_{h} v^{n}\right)\left|\nabla u^{n}\right|\right\|^{2} \\
& +\left\{(2 \epsilon)^{-1}\left\langle v^{n}-1, v^{n}-v^{n-1}\right\rangle+2 \epsilon\left\langle\nabla v^{n}, \nabla v^{n}-\nabla v^{n-1}\right\rangle\right\} .
\end{aligned}
$$

Writing $v^{n}-v^{n-1}=\left(v^{n}-1\right)-\left(v^{n-1}-1\right)$ in the second term on the right-hand side, and performing calculations in the term in the curly brackets similar to the ones employed in (3.7) we obtain

$$
\begin{aligned}
-\frac{1}{2} \int_{\Omega}\left(a^{n}-a^{n-1}\right)\left|\nabla u^{n}\right|^{2} \mathrm{~d} x= & \mathcal{H}\left(v^{n}\right)-\mathcal{H}\left(v^{n-1}\right) \\
& +h^{2}\left((4 \epsilon)^{-1}\left\|\delta_{h} v^{n}\right\|^{2}+\epsilon\left\|\nabla \delta_{h} v^{n}\right\|^{2}\right)+\frac{1}{2} h^{2}\left\|\left(\delta_{h} v^{n}\right) \nabla u^{n}\right\|^{2} .
\end{aligned}
$$


Hence, summing (3.6) over $n$ and using (3.7)-(3.10) we arrive at

$$
\begin{aligned}
{\left[\mathcal{K}\left(\delta_{h} u^{N}\right)+\mathcal{E}\left(u^{N}, v^{N}\right)+\mathcal{H}\left(v^{N}\right)\right]-\left[\mathcal{K}\left(\delta_{h} u_{h}^{0}\right)+\mathcal{E}\right.} & \left.\left(u^{0}, v^{0}\right)+\mathcal{H}\left(v^{0}\right)\right] \\
& +\sum_{n=1}^{N}\left\|\left(a^{n-1}\right)^{1 / 2} \nabla \delta_{h} u^{n}\right\|^{2}+h \sum_{n=1}^{N} h D_{h}^{n}=\sum_{n=1}^{N} I_{h}^{n}
\end{aligned}
$$

where

$$
\begin{aligned}
D_{h}^{n}= & \frac{1}{2}\left\|\delta_{h}^{2} u^{n}\right\|^{2}+\frac{1}{2}\left\|\left(a^{n-1}\right)^{1 / 2} \nabla \delta_{h} u^{n}\right\|^{2}+\frac{1}{2}\left\|\delta_{h} v^{n} \nabla u^{n}\right\| \\
& +\frac{1}{4 \gamma}\left\|\delta_{h} v^{n}\right\|^{2}+\gamma\left\|\nabla \delta_{h} v^{n}\right\|^{2}
\end{aligned}
$$

and

$$
I_{h}^{n}=h\left\langle\delta_{h}^{2} u^{n}, \delta_{h} g^{n}\right\rangle+h\left\langle a^{n-1} \nabla\left(u^{n}+\delta_{h} u^{n}\right), \nabla \delta_{h} g^{n}\right\rangle .
$$

We next observe that

$$
\sum_{n=1}^{N} h\left\langle\delta_{h}^{2} u^{n}, \delta_{h} g^{n}\right\rangle=\left\langle\delta_{h} u^{N}, \delta_{h} g^{N}\right\rangle-\left\langle\delta_{h} u^{0}, \delta_{h} g^{1}\right\rangle-\sum_{n=1}^{N-1} h\left\langle\delta_{h} u^{n}, \delta_{h}^{2} g^{n+1}\right\rangle
$$

and hence from a few $\epsilon$-Cauchy's inequalities with $\epsilon_{i}>0, i \in\{1,2,3,4\}$, and using the bound $\eta_{\gamma} \leq\left\|a_{h}^{n}\right\|_{\infty} \leq$ $1+\eta_{\gamma}$ we have

$$
\begin{aligned}
\left|\sum_{n=1}^{N} I_{h}^{n}\right| \leq & \epsilon_{1}\left\|\delta_{h} u^{N}\right\|^{2}+\frac{1}{4 \epsilon_{1}}\left\|\delta_{h} g^{N}\right\|^{2}+\left|\left\langle\delta_{h} u^{0}, \delta_{h} g^{1}\right\rangle\right|+\epsilon_{2} \sum_{n=1}^{N-1} h\left\|\delta_{h} u^{n}\right\|^{2} \\
& +\frac{1}{4 \epsilon_{2}} \sum_{n=1}^{N-1} h\left\|\delta_{h}^{2} g^{n+1}\right\|^{2}+\epsilon_{3}\left(1+\eta_{\gamma}\right) \sum_{n=1}^{N} h\left\|\nabla u^{n}\right\|^{2} \\
& +\frac{1+\eta_{\gamma}}{4 \epsilon_{3}} \sum_{n=1}^{N} h\left\|\nabla \delta_{h} g^{n}\right\|^{2}+\epsilon_{4} \sum_{n=1}^{N} h\left\|\left(a^{n-1}\right)^{1 / 2} \nabla \delta_{h} u^{n}\right\|^{2}+\frac{1+\eta_{\gamma}}{4 \epsilon_{4}} \sum_{n=1}^{N} h\left\|\nabla \delta_{h} g^{n}\right\|^{2} .
\end{aligned}
$$

Finally, we observe that $\sum_{n=1}^{N-1} h\left\|\delta_{h} u^{n}\right\|^{2} \leq T_{f} \max _{n}\left\|\delta_{h} u^{n}\right\|^{2}$ and $\sum_{n=1}^{N} h\left\|\nabla u^{n}\right\|^{2} \leq T_{f} \max _{n}\left\|\nabla u^{n}\right\|^{2}$. Choosing $\epsilon_{i}$ sufficiently small, using the coercivity of the different energies, and the fact that $D_{h}^{n} \geq 0$ we obtain (3.3) from (3.11).

We observe that the RHS of the a priori estimate (3.3), when applied to obtain bounds for $u_{\epsilon}$, and $v_{\epsilon}$ also depends on $\epsilon$, through $g_{\epsilon}$ and $T_{f}=1 / \epsilon$ (see (3.5)). The next lemma provides a bound for $u^{\epsilon}$ and $v^{\epsilon}$ by estimating $I\left(g_{\epsilon}, \rho_{0}, v_{0}, \epsilon \rho_{1}\right)$ independently of $\epsilon$.

Lemma 3.3. Let $u^{\epsilon}$ and $a^{\epsilon}$ be as in Definition 2.3, and assume $g \in H^{2}\left(0,1 ; H^{1}\right)$. Then,

$$
\left\|\nabla u^{\epsilon}\right\|_{L^{\infty}\left(0,1 ; L^{2}\right)} \leq c, \text { and }\left\|v^{\epsilon}\right\|_{L^{\infty}\left(0,1 ; H^{1}\right)} \leq c
$$

and

$$
\left\|\dot{u}^{\epsilon}\right\|_{L^{\infty}\left(0,1 ; L^{2}\right)} \leq \frac{c}{\epsilon}, \quad \text { and }\left\|\dot{u}^{\epsilon}\right\|_{L^{2}\left(0,1 ; H^{1}\right)} \leq \frac{c}{\sqrt{\epsilon}} .
$$


Proof. Recalling that $g_{\epsilon}(t)=g(\epsilon t)$ and $s=\epsilon t$, we observe that if $g \in H^{2}\left(0,1 ; H^{1}\right)$, then as $h \rightarrow 0$ we have (see for instance Lem. 3.9 from [16])

$$
\begin{aligned}
\sum_{n=1}^{N_{f}-1} h\left\|\delta_{h}^{2} g_{\epsilon}^{n+1}\right\|^{2} & \rightarrow \int_{0}^{1 / \epsilon}\left\|\partial_{t}^{2} g_{\epsilon}(t)\right\|^{2} \mathrm{~d} t=\int_{0}^{1} \epsilon^{3}\left\|g_{s s}\right\|^{2} \mathrm{~d} s, \\
\sum_{n=1}^{N_{f}} h\left\|\nabla \delta_{h} g_{\epsilon}^{n}\right\|^{2} & \rightarrow \int_{0}^{1 / \epsilon}\left\|\nabla \partial_{t} g_{\epsilon}(t)\right\|^{2} \mathrm{~d} t=\int_{0}^{1} \epsilon\left\|\nabla g_{s}\right\|^{2} \mathrm{~d} s .
\end{aligned}
$$

We also have that

$$
\delta_{h} u^{0}=\epsilon \rho_{1} \quad \text { and } \delta_{h} g_{\epsilon}^{1} \rightarrow \epsilon g_{s}(0)
$$

Hence, from (3.4), we obtain that

$$
\begin{aligned}
\underset{h}{\limsup I\left(g_{\epsilon}, \rho_{0}, v_{0}, \epsilon \rho_{1}\right) \leq} & \mathcal{F}\left(\rho_{0}, v_{0}, \epsilon \rho_{1}\right)+\epsilon^{2}\left\|\rho_{1}\right\|\left\|g_{s}(0)\right\|+c \frac{1}{\epsilon} \int_{0}^{1} \epsilon^{3}\left\|g_{s s}\right\|^{2} \mathrm{~d} s \\
& +c\left(1+\frac{1}{\epsilon}\right) \int_{0}^{1} \epsilon\left\|\nabla g_{s}\right\|^{2} \mathrm{~d} s+c
\end{aligned}
$$

to conclude

$$
I\left(g_{\epsilon}, \rho_{0}, v_{0}, \epsilon \rho_{1}\right) \leq c
$$

with $c$ independent of $\epsilon$. Hence, recalling that $D_{h}^{n}>0$ and $a^{n-1} \geq \eta_{\gamma}$ the a priori estimate (3.3) yields

$$
\begin{array}{r}
\left\|u_{\epsilon}\right\|_{W^{1, \infty}\left(0,1 / \epsilon ; L^{2}\right)} \leq c, \quad\left\|u_{\epsilon}\right\|_{L^{\infty}\left(0,1 / \epsilon ; H^{1}\right)} \leq c \\
\left\|v_{\epsilon}\right\|_{L^{\infty}\left(0,1 / \epsilon ; H^{1}\right)} \leq c \int_{0}^{1 / \epsilon}\left\|\partial_{t} u_{\epsilon}(t)\right\|_{1}^{2} \mathrm{~d} t \leq c .
\end{array}
$$

We obtain the desired result using that $(s=\epsilon t)$

$$
\int_{0}^{1 / \epsilon}\left\|\partial_{t} u_{\epsilon}(t)\right\|_{1}^{2} \mathrm{~d} t=\int_{0}^{1} \epsilon\left\|\partial_{s} u^{\epsilon}(s)\right\|_{1}^{2} \mathrm{~d} s
$$

The last lemma yields the existence a subsequence $\left\{\epsilon_{j}\right\}$, which we still denote $\{\epsilon\}$, and functions $u^{*}, v^{*} \in$ $L^{2}\left(0,1 ; H^{1}\right)$ such that

\section{Definition 3.4.}

$$
u^{\epsilon} \rightarrow u^{*} \text { and } v^{\epsilon} \rightarrow v^{*} \text { in } L^{2}\left(0,1 ; H^{1}\right)
$$

as $\epsilon \rightarrow 0$. We also define

$$
a^{*}(s)=v^{*}(s)^{2}+\eta_{\gamma}
$$

\section{Conservation of energy}

In this section we study when $u^{*}$ and $v^{*}$ obtained in the last section satisfy the conservation of energy from the limit regularized quasi-static model (item ( $\tilde{\mathrm{d}})$ in Sect. 2.4). The main result of this section is Theorem 4.7, which provides sufficient conditions on $u^{\epsilon}$ and $v^{\epsilon}$ such that $u^{*}$ and $v^{*}$ satisfy the quasi-static conservation of energy. The hypotheses employed to guarantee this result are

$$
v^{\epsilon} \rightarrow v^{*} \text { in } L^{2}\left(0,1 ; H^{1}\right), \quad u^{\epsilon} \rightarrow u^{*} \text { in } L^{2}\left(0,1 ; H^{1}\right)
$$

and the important condition

$$
\partial_{s} v^{\epsilon} \in L^{2}\left([0,1] ; L^{\infty}\right)
$$


This last assumption has a physical meaning. It imposes a restriction on the speed of fracture propagation for the solution of the dynamic problem $\partial_{t} v_{\epsilon}$ with boundary condition given by $g_{\epsilon}(t)=g(\epsilon t)$. Formally, it says that the speed of crack propagation of the dynamical model should slow down following the deceleration of boundary displacement. More precisely, the last hypothesis is satisfied, for instance, if there exists a function $\omega \in H^{1}([0,1])$ and a subsequence $\left\{\epsilon_{j}\right\}$ (still denoted $\{\epsilon\}$ ) employed in the definition of $v^{*}(3.19)$ such that the maximum speed of fracture propagation is bounded as follows

$$
\left\|\partial_{t} v_{\epsilon}(t)\right\|_{L^{\infty}(\Omega)} \leq\left|\partial_{t} \omega_{\epsilon}(t)\right| \text {, a.e. } t \in[0,1 / \epsilon] \quad \text { with } \omega_{\epsilon}(t)=\omega(\epsilon t) .
$$

Then

$$
\begin{aligned}
\int_{0}^{1}\left\|\dot{v}^{\epsilon}(s)\right\|_{L^{\infty}}^{2} \mathrm{~d} s & \leq c \int_{0}^{1 / \epsilon}\left\|\partial_{t} v_{\epsilon}(t)\right\|_{L^{\infty}}^{2} \frac{\mathrm{d} t}{\epsilon}, \quad\left(v^{\epsilon}(s)=v_{\epsilon}(s / \epsilon), t=s / \epsilon\right) \\
& \leq c \int_{0}^{1 / \epsilon}\left|\partial_{t} \omega_{\epsilon}(t)\right|^{2} \frac{\mathrm{d} t}{\epsilon} \leq c\|\omega\|_{H^{1}([0,1])}^{2} .
\end{aligned}
$$

We now present some lemmas used in the proof of Theorem 4.7.

Let $u$ be the solution of a linear second order elliptic PDE's depending on a parameter $s$ through the boundary condition and the coefficients of the equation. The following lemma shows the smooth dependence of $u$ with respect to the parameter $s$, and it is an important tool in the proof of our main result.

Lemma 4.1. Let $a(s):[0,1] \rightarrow L^{\infty}(\Omega)$ be such that $c_{0}<a(s, x)<c_{1} \quad \forall(s, x) \in[0,1] \times \Omega$ for constants $0<c_{0}<c_{1}$. Let $g \in H^{1}\left(0, T ; H^{1}\right)$, and assume

$$
\|a\|_{H^{1}\left(0,1 ; L^{\infty}\right)} \leq c
$$

for a constant $c>0$. For $s \in[0,1]$ set $u(s) \in H^{1}(\Omega)$ as the weak solution of

$$
\nabla \cdot a(s) \nabla u(s)=0 \quad \text { in } \Omega, \quad \text { and }\left.u(s)\right|_{\partial \Omega}=\left.g(s)\right|_{\partial \Omega} .
$$

Then,

$$
u \in H^{1}\left(0,1 ; H^{1}\right) .
$$

Proof. We first observe $c_{0}<a(s, x)<c_{1}$ and the definition of $u$ yield

$$
|u(s)|_{1}^{2} \leq c \int_{\Omega} a(s)|\nabla u(s)|^{2} \mathrm{~d} x \leq c|g(s)|_{1}^{2} .
$$

Hence, from a Poincare inequality and the fact that $g \in H^{1}\left(0, T ; H^{1}\right)$ implies $g \in L^{\infty}\left(0,1 ; H^{1}\right)$ we obtain

$$
u \in L^{\infty}\left(0,1 ; H^{1}\right) .
$$

Let $h>0$, and given a function $z:[0, T] \rightarrow L^{2}(\Omega)$ define $\delta z(s)=z(s+h)-z(s)$

$$
\begin{aligned}
c\|\nabla \delta u(s)\|^{2} \leq & \langle a(s) \nabla \delta u(s), \nabla \delta u(s)\rangle \\
\leq & \langle a(s) \nabla u(s+h), \nabla \delta u(s)\rangle-\langle a(s) \nabla u(s), \nabla \delta u(s)\rangle \\
& +\langle a(s) \nabla u(s), \nabla(\delta u(s)-\delta g(s))\rangle-\langle a(s+h) \nabla u(s+h), \nabla(\delta u(s)-\delta g(s))\rangle
\end{aligned}
$$

where we have used the fact that $\langle a(s) \nabla(u(s)), \nabla \phi\rangle=0$ for all $\phi \in H_{0}^{1}(\Omega)$, and hence the two last terms on the RHS of the last inequality are equal to zero. By a simple calculation we obtain

$$
\begin{aligned}
c\|\nabla \delta u(s)\|^{2} \leq & -\langle\delta a(s) \nabla u(s+h), \nabla \delta u(s)\rangle \\
& +\langle a(s+h) \nabla u(s+h), \nabla \delta g(s)\rangle-\langle a(s) \nabla u(s), \nabla \delta g(s)\rangle .
\end{aligned}
$$


Summing and subtracting $\langle a(s+h) \nabla u(s), \nabla \delta g(s)\rangle$ and rearranging the terms we obtain

$$
\begin{aligned}
c\|\nabla \delta u(s)\|^{2} \leq & -\langle\delta a(s) \nabla u(s+h), \nabla \delta u(s)\rangle+\langle a(s+h) \nabla \delta u(s), \nabla \delta g(s)\rangle \\
& +\langle\delta a(s) \nabla u(s), \nabla \delta g(s)\rangle .
\end{aligned}
$$

We now use an $\epsilon$-Cauchy's inequality to obtain

$$
\begin{gathered}
c\|\nabla \delta u(s)\|^{2} \leq \\
+\|\delta a(s) \nabla u(s+h)\|^{2}+\|\nabla \delta g(s)\|^{2} \\
+\|\delta a(s) \nabla u(s)\|\|\nabla \delta g(s)\| .
\end{gathered}
$$

Dividing both sides of the last inequality by $h^{2}$ and using $g \in H^{1}\left(0, T ; H^{1}\right)$, (4.3), and (4.6) we obtain for sufficiently small $0<h$

$$
\int_{0}^{1-h} \int_{\Omega}\left|\frac{\nabla(u(s+h)-u(s))}{h}\right|^{2} \mathrm{~d} x \mathrm{~d} s \leq c .
$$

The definition of the weak derivative yields the desired result.

The following lemma shows under appropriate conditions that $u^{*}(s)$ minimizes the elastic energy associated to a crack configuration represented by $v^{*}(s)$.

Lemma 4.2. Let $u^{\epsilon}$ and $v^{\epsilon}$ be as in Definition 2.3, and $u^{*}$ and $v^{*}$ be their respective weak limits provided by a subsequence $\epsilon \rightarrow 0$ as in (3.19). Assume

$$
a^{\epsilon} \rightarrow a^{*} \text { a.e. in }[0, T] \times \Omega \text { when } \epsilon \rightarrow 0 .
$$

Then $u^{*}=u$ in the $L^{2}\left(0,1 ; H^{1}\right)$ topology, where $u(s)$ is the weak solution of

$$
\nabla \cdot\left(a^{*}(s) \nabla u(s)\right)=0 \text { in } \Omega \text { and } u(s)=g(s) \text { on } \partial \Omega \text {, for } s \in[0,1] .
$$

Proof. Recall from Theorem 2.1 that $u^{\epsilon} \in H^{2}\left(0,1 ; H^{1}\right)$, and let $\phi \in C^{2}\left(0,1 ; H_{0}^{1}\right)$ and $S \in[0,1]$, from a Cauchy's inequality, the second a priori estimate in (3.15), and the fact that $0 \leq\left|a^{\epsilon}\right| \leq 1$ we have

$$
\epsilon \int_{0}^{S}\left|\left\langle a^{\epsilon} \nabla \dot{u}^{\epsilon}, \nabla \phi\right\rangle\right| \mathrm{d} s \leq \epsilon\left\|\dot{u}^{\epsilon}\right\|_{L^{2}\left(0,1 ; H^{1}\right)}\|\phi\|_{L^{2}\left(0,1 ; H^{1}\right)} \leq \sqrt{\epsilon} c\|\phi\|_{L^{2}\left(0,1 ; H^{1}\right)} .
$$

Hence,

$$
\lim _{\epsilon \rightarrow 0} \epsilon \int_{0}^{S}\left\langle a^{\epsilon} \nabla \dot{u}^{\epsilon}, \nabla \phi\right\rangle \mathrm{d} s=0, \forall \phi \in C\left(0,1 ; H_{0}^{1}\right) \quad \text { for } S \in[0,1]
$$

Taking $\varphi=\phi(s)$ in (2.11) and integrating by parts in time we obtain

$$
\begin{aligned}
\int_{0}^{S} \epsilon^{2}\left\langle\ddot{u}^{\epsilon}, \phi\right\rangle+\left\langle a^{\epsilon} \nabla\left(u^{\epsilon}+\epsilon \dot{u}^{\epsilon}\right), \nabla \phi\right\rangle \mathrm{d} s= & \epsilon^{2}\left\langle\dot{u}^{\epsilon}(S), \phi(S)\right\rangle-\epsilon^{2}\left\langle\dot{u}^{\epsilon}(0), \phi(0)\right\rangle \\
& -\int_{0}^{S} \epsilon^{2}\left\langle\dot{u}^{\epsilon}, \dot{\phi}\right\rangle-\left\langle a^{\epsilon} \nabla u^{\epsilon}, \nabla \phi\right\rangle \mathrm{d} s+\epsilon \int_{0}^{S}\left\langle a^{\epsilon} \nabla \dot{u}^{\epsilon}, \nabla \phi\right\rangle \mathrm{d} s=0 .
\end{aligned}
$$

We use estimate (3.15) to conclude that as $\epsilon \rightarrow 0$

$$
\epsilon^{2}\left\langle\dot{u}^{\epsilon}(S), \phi(S)\right\rangle-\epsilon^{2}\left\langle\dot{u}^{\epsilon}(0), \phi(0)\right\rangle-\int_{0}^{S} \epsilon^{2}\left\langle\dot{u}^{\epsilon}, \dot{\phi}\right\rangle \mathrm{d} s \rightarrow 0 .
$$

Therefore, (4.11) and (4.12) yield

$$
\int_{0}^{S}\left\langle a^{\epsilon} \nabla u^{\epsilon}, \nabla \phi\right\rangle \mathrm{d} s \rightarrow 0
$$


We now observe that for all $\phi \in C^{2}\left(0,1 ; H_{0}^{1}\right)$

$$
\int_{0}^{S}\left\langle a^{*} \nabla u^{*}, \nabla \phi\right\rangle-\left\langle a^{\epsilon} \nabla u^{\epsilon}, \nabla \phi\right\rangle \mathrm{d} s=\int_{0}^{S}\left\langle\nabla u^{*}, a^{*} \nabla \phi\right\rangle-\left\langle\nabla u^{\epsilon}, a^{\epsilon} \nabla \phi\right\rangle \mathrm{d} s .
$$

Using the fact that for $a^{\epsilon} \nabla \phi \rightarrow a^{*} \nabla \phi$ a.e. in $[0, T] \times \Omega$, and that $a^{\epsilon}$ and $a^{*}$ are uniformly bounded in $L^{\infty}([0,1] \times \Omega)$, we obtain from the Dominated convergence theorem that $a^{\epsilon} \nabla \phi \rightarrow a^{*} \nabla \phi$ in $L^{2}\left(0, T ; L^{2}(\Omega)^{n}\right)$. Finally, using the weak convergence of $u^{\epsilon}$ to $u^{*}$ in $L^{2}\left(0,1 ; H^{1}\right)$ we conclude that

$$
\int_{0}^{S}\left\langle a^{*} \nabla u^{*}, \nabla \phi\right\rangle \mathrm{d} s=\lim _{\epsilon \rightarrow 0} \int_{0}^{S}\left\langle a^{\epsilon} \nabla u^{\epsilon}, \nabla \phi\right\rangle \mathrm{d} s=0,
$$

yielding (4.10).

Remark 4.3. We have that $u^{*}=u$ in the $L^{2}\left(0,1 ; H^{1}\right)$ topology. Hence, we redefine (if necessary) $u^{*}$ in a set of measure zero in $[0,1]$ such that $u^{*}(s)=u(s)$ for all $s \in[0,1]$.

Remark 4.4. From the Dominated convergence theorem we conclude that (4.9) is equivalent to

$$
a^{\epsilon} \rightarrow a^{*} \text { in } L^{2}([0, T] \times \Omega) .
$$

The following auxiliary lemma is used to obtain our main result. It relates the convergence of the functionals $\mathcal{H}\left(v^{\epsilon}\right)$ with the strong convergence of $v^{\epsilon}$ to $v^{*}$ in $L^{2}\left(0,1 ; H^{1}\right)$.

Lemma 4.5. Let $v^{\epsilon}$ and $v^{*}$ be defined by (2.10) and (3.19), respectively. Then

$$
\mathcal{H}\left(v^{\epsilon}(s)\right) \rightarrow \mathcal{H}\left(v^{*}(s)\right) \text { for } s \text { a.e. in }[0,1] \text { when } \epsilon \rightarrow 0
$$

if and only if

$$
v^{\epsilon} \rightarrow v^{*} \quad \text { in } \quad L^{2}\left(0,1 ; H^{1}\right) \quad \text { when } \epsilon \rightarrow 0 .
$$

Proof. $(\Rightarrow)$ : We first define the norm

$$
\|\phi\|_{\mathcal{H}}^{2}=\int_{\Omega} \frac{1}{4 \gamma} \phi^{2}+\gamma|\nabla \phi|^{2} \mathrm{~d} x
$$

which is equivalent to the $H^{1}$ norm for fixed $\gamma$, and has the following property

$$
\|1-\phi\|_{\mathcal{H}}^{2}=\mathcal{H}(\phi)
$$

Therefore, denoting $V$ the space $H^{1}$ equipped with the $\|\cdot\|_{\mathcal{H}}$ norm, we obtain from (3.14) that up to a subsequence

$$
1-v^{\epsilon} \rightarrow 1-v^{*} \text { in } L^{2}(0,1 ; V) .
$$

If (4.16) holds then $\left\|1-v^{\epsilon}(s)\right\|_{\mathcal{H}} \rightarrow\left\|1-v^{*}(s)\right\|_{\mathcal{H}}$ for $s$ a.e. in $[0,1]$. Hence, by (3.14) and the dominated convergence theorem we have

$$
\left\|1-v^{\epsilon}\right\|_{L^{2}(0,1 ; V)} \rightarrow\left\|1-v^{*}\right\|_{L^{2}(0,1 ; V)}
$$

and therefore $1-v^{\epsilon} \rightarrow 1-v^{*}$ in $L^{2}(0,1 ; V)$, yielding (4.17).

$(\Leftarrow)$ : The proof of the other direction of the equivalence is straightforward.

The next proposition provides sufficient conditions to obtain that the functions $u^{*}$ and $v^{*}$ satisfy the quasistatic conservation of energy (2.3). 
Proposition 4.6. Let $u^{\epsilon}$ and $v^{\epsilon}$ be as in Definition 2.3, and $u^{*}$ and $v^{*}$ be their respective weak limits provided by a subsequence $\epsilon \rightarrow 0$ given by (3.19) and Remark 4.3. Assume

$$
\left\|a^{\epsilon}\right\|_{H^{1}\left(0,1 ; L^{\infty}\right)} \leq c
$$

and

$$
v^{\epsilon} \rightarrow v^{*} \quad \text { in } L^{2}\left(0,1 ; H^{1}\right) \text {, and } u^{\epsilon} \rightarrow u^{*} \text { in } L^{2}\left(0,1 ; H^{1}\right) .
$$

Then the pair $\left(u^{*}, v^{*}\right)$ satisfies the quasi-static conservation of energy equation (2.3).

Proof. Hypothesis (4.19) yields $a^{*} \in H^{1}\left(0,1 ; L^{\infty}\right)$, and from $(4.20)$ and Lemmas 4.2 and 4.1 we conclude that $u^{*}$ is the solution of $(4.10)$ and $u^{*} \in H^{1}\left(0,1 ; H^{1}\right)$. Hence, the following derivation rule holds

$$
\begin{aligned}
\mathcal{E}\left(u^{*}(S), v^{*}(S)\right)-\mathcal{E}\left(u^{*}(0), v^{*}(0)\right) & =\int_{0}^{S} \frac{d \mathcal{E}\left(u^{*}, v^{*}\right)}{\mathrm{d} s} \mathrm{~d} s \\
& =\int_{0}^{S} \frac{\left\langle\dot{a}^{*} \nabla u^{*}, \nabla u^{*}\right\rangle}{2}+\left\langle a^{*} \nabla u^{*}, \nabla \dot{u}^{*}\right\rangle \mathrm{d} s .
\end{aligned}
$$

Recall the notation $\delta_{h} g(s)=(g(s+h)-g(s)) / h$. Since $u^{*}$ and $g \in H^{1}\left(0,1 ; H^{1}\right)$ we have that $\delta_{h} g$ and $\delta_{h} u^{*}$ weakly converge to $\dot{g}$ and $\dot{u}^{*}$ in $L^{2}\left(0, T ; H^{1}\right)$, respectively. From (4.10) and the fact that $\delta_{h} g-\delta_{h} u^{*} \in L^{2}\left(0, T ; H_{0}^{1}\right)$ $\left(\left.u^{*}(s)\right|_{\partial \Omega}=\left.g(s)\right|_{\partial \Omega}\right.$ for $\left.s \in[0,1]\right)$ we have

$$
\int_{0}^{S}\left\langle a^{*} \nabla u^{*}, \nabla \dot{u}^{*}\right\rangle \mathrm{d} s=\int_{0}^{S}\left\langle a^{*} \nabla u^{*}, \nabla \dot{g}\right\rangle \mathrm{d} s
$$

Therefore, $u^{*}$ and $v^{*}$ satisfy the conservation of energy equation (2.3) if

$$
\mathcal{E}\left(u^{*}(S), v^{*}(S)\right)+\mathcal{H}\left(v^{*}(S)\right)=\mathcal{E}\left(u^{*}(0), v^{*}(0)\right)+\mathcal{H}\left(v^{*}(0)\right)+\int_{0}^{S}\left\langle a^{*} \nabla u^{*}, \nabla \dot{u}^{*}\right\rangle \mathrm{d} s .
$$

Hence, from (4.21) the pair $\left(u^{*}, v^{*}\right)$ satisfies the conservation of energy equation (2.3) if

$$
\int_{0}^{S} \frac{\left\langle\dot{a}^{*} \nabla u^{*}, \nabla u^{*}\right\rangle}{2} \mathrm{~d} s+\mathcal{H}\left(v^{*}(S)\right)-\mathcal{H}\left(v^{*}(0)\right)=0 .
$$

We observe that $\dot{a}^{\epsilon} \rightarrow \dot{a}^{*}$ in the $L^{2}\left(0,1 ; L^{\infty}\right)$ weak-* topology, and $u^{\epsilon} \rightarrow u^{*}$ in $L^{2}\left(0,1 ; H^{1}\right)$ when $\epsilon \rightarrow 0$, hence

$$
\int_{0}^{S}\left\langle\dot{a}^{\epsilon} \nabla u^{\epsilon}, \nabla u^{\epsilon}\right\rangle \mathrm{d} s \rightarrow \int_{0}^{S}\left\langle\dot{a}^{*} \nabla u^{*}, \nabla u^{*}\right\rangle \mathrm{d} s .
$$

An argument similar to the one used to obtain (4.22), and equation (2.11) with $\varphi=\dot{u}_{\epsilon}-\dot{g}_{\epsilon} \in L^{2}\left(0,1 ; H_{0}^{1}\right)$ yield

$$
\int_{0}^{S} \epsilon^{2}\left\langle\ddot{u}^{\epsilon}, \dot{u}^{\epsilon}-\dot{g}\right\rangle \mathrm{d} s+\int_{0}^{S}\left\langle a^{\epsilon} \nabla\left(u^{\epsilon}+\epsilon \dot{u}^{\epsilon}\right), \nabla\left(\dot{u}^{\epsilon}-\dot{g}\right)\right\rangle \mathrm{d} s=0 .
$$

From (2.13) we have

$$
\begin{aligned}
\mathcal{E}\left(u^{\epsilon}(S), v^{\epsilon}(S)\right)+\mathcal{H}\left(v^{\epsilon}(S)\right)+\epsilon^{2} \mathcal{K}\left(\dot{u}^{\epsilon}(S)\right)= & \mathcal{E}\left(u^{\epsilon}(0), v^{\epsilon}(0)\right)+\mathcal{H}\left(v^{\epsilon}(0)\right) \\
& +\epsilon^{2} \mathcal{K}\left(\dot{u}^{\epsilon}(0)\right)-\int_{0}^{S} \epsilon\left\|\sqrt{a^{\epsilon}} \nabla \dot{u}^{\epsilon}\right\|^{2} \mathrm{~d} s \\
& +\int_{0}^{S} \epsilon^{2}\left\langle\ddot{u}^{\epsilon}, \dot{u}^{\epsilon}\right\rangle+\left\langle a^{\epsilon} \nabla\left(u^{\epsilon}+\epsilon \dot{u}^{\epsilon}\right), \nabla \dot{u}^{\epsilon}\right\rangle \mathrm{d} s .
\end{aligned}
$$


From (4.19) and Lemma 3.3 we obtain enough regularity, $u^{\epsilon} \in H^{2}\left(0,1 ; L^{2}\right)$, to guarantee the following derivation rules

$$
\begin{gathered}
\mathcal{K}\left(\dot{u}^{\epsilon}(S)\right)-\mathcal{K}\left(\dot{u}^{\epsilon}(0)\right)=\int_{0}^{S}\left\langle\ddot{u}^{\epsilon}, \dot{u}^{\epsilon}\right\rangle \mathrm{d} s \\
\mathcal{E}\left(u^{\epsilon}(S), v^{\epsilon}(S)\right)-\mathcal{E}\left(u^{\epsilon}(0), v^{\epsilon}(0)\right)=\int_{0}^{S} \frac{1}{2}\left\langle\dot{a}^{\epsilon} \nabla u^{\epsilon}, u^{\epsilon}\right\rangle+\left\langle a^{\epsilon} \nabla u^{\epsilon}, \nabla \dot{u}^{\epsilon}\right\rangle \mathrm{d} s,
\end{gathered}
$$

and hence

$$
\int_{0}^{S} \frac{\left\langle\dot{a}^{\epsilon} \nabla u^{\epsilon}, \nabla u^{\epsilon}\right\rangle}{2} \mathrm{~d} s+\mathcal{H}\left(v^{\epsilon}(S)\right)-\mathcal{H}\left(v^{\epsilon}(0)\right)=0 .
$$

Therefore, taking the limit $\epsilon \rightarrow 0$ in the last equation, using (4.24) and Lemma 4.5 we obtain (4.23), and the desired result follows.

We now observe that

$$
\dot{a}^{\epsilon}(s)=2 v^{\epsilon}(s) \dot{v}^{\epsilon}(s)
$$

and since $0 \leq v^{\epsilon} \leq 1$ we have

$$
\left\|\dot{a}^{\epsilon}\right\|_{L^{2}\left([0,1], L^{\infty}\right)} \leq 2\left\|\dot{v}^{\epsilon}\right\|_{L^{2}\left([0,1], L^{\infty}\right)} .
$$

Hence, the last estimate combined with Proposition 4.6 yield the following result.

Theorem 4.7. Let $g \in H^{2}\left(0,1 ; H^{1}\right)$, $u^{\epsilon}$ and $v^{\epsilon}$ be as in Definition 2.3, and $u^{*}$ and $v^{*}$ be their respective weak limits provided by a subsequence $\epsilon \rightarrow 0$ as in (3.19) and Remark 4.3. Assume (4.1) is satisfied and

$$
v^{\epsilon} \rightarrow v^{*} \text { in } L^{2}\left(0,1 ; H^{1}\right) \text {, and } u^{\epsilon} \rightarrow u^{*} \text { in } L^{2}\left(0,1 ; H^{1}\right) .
$$

Then $u^{*}$ and $v^{*}$ satisfy the quasi-static conservation of energy (2.3). More precisely,

$$
\begin{aligned}
\mathcal{E}\left(u^{*}(S), v^{*}(S)\right)+\mathcal{H}\left(v^{*}(S)\right)= & \mathcal{E}\left(u^{*}(0), v^{*}(0)\right)+\mathcal{H}(v(0)) \\
& +\int_{0}^{S}\left\langle\left(\eta_{\gamma}+\left(v^{*}\right)^{2}\right) \nabla u^{*}, \nabla \dot{g}\right\rangle \mathrm{d} s .
\end{aligned}
$$

\section{Minimality CONDition}

In this section we derive conditions such that the pair $\left(u^{*}, v^{*}\right)$ satisfies the minimality condition from item

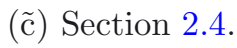

Lemma 5.1. Let $g \in H^{2}\left(0,1 ; H^{1}\right), u^{\epsilon}$ and $v^{\epsilon}$ be as in Definition 2.3, and $u^{*}$ and $v^{*}$ be their respective weak limits provided by subsequence $\epsilon \rightarrow 0$ as in (3.19) and Remark 4.3. Also, assume

$$
u^{\epsilon} \rightarrow u^{*} \text { in } L^{2}\left(0,1 ; H^{1}\right)
$$

and

$$
v^{\epsilon} \rightarrow v^{*} \text { in } L^{\infty}([0,1] \times \Omega) \cap L^{2}\left(0,1 ; H^{1}\right)
$$

Then, for every $s \in[0,1]$

$$
\mathcal{E}\left(u^{*}(s), v^{*}(s)\right)+\mathcal{H}\left(v^{*}(s)\right)=\inf _{z \leq v^{*}(s)} \mathcal{E}\left(u^{*}(s), z\right)+\mathcal{H}(z),
$$

and

$$
\mathcal{E}\left(u^{*}(s), v^{*}(s)\right)+\mathcal{H}\left(v^{*}(s)\right)=\inf _{\phi-g \in H_{0}^{1}(\Omega)} \mathcal{E}\left(\phi, v^{*}(s)\right)+\mathcal{H}\left(v^{*}(s)\right)
$$

Proof. Equation (5.1) follows from the strong convergence hypotheses, and the fact that $v^{\epsilon}$, and $u^{\epsilon}$ satisfy (2.12). Lemma 4.2 yields (5.2). 


\section{Convergence to A QuAsi-StatiC Evolution}

In this section we combine Theorem 4.7 and Lemma 5.1 to obtain conditions guaranteing the convergence of $u^{\epsilon}$, and $v^{\epsilon}$ to functions satisfying the alternative quasi-static model from Section 2.4.

Theorem 6.1. Let $g \in H^{2}\left(0,1 ; H^{1}\right)$, $u^{\epsilon}$ and $v^{\epsilon}$ be as in Definition 2.3, and $u^{*}$ and $v^{*}$ be their respective weak limits provided by a subsequence $\epsilon \rightarrow 0$ as in (3.19) and Remark 4.3. Assume (4.1) is satisfied and

$$
u^{\epsilon} \rightarrow u^{*} \text { in } L^{2}\left(0,1 ; H^{1}\right)
$$

and

$$
v^{\epsilon} \rightarrow v^{*} \text { in } L^{\infty}([0,1] \times \Omega) \cap L^{2}\left(0,1 ; H^{1}\right)
$$

Then the pair $\left(u^{*}, v^{*}\right)$ is a solution of the limit regularized quasi-static model proposed in Section 2.4.

Proof. Direct consequence of Theorem 4.7 and Lemma 5.1.

Acknowledgements. The author is thankful to Prof. Christopher Larsen for bringing this problem to his attention and some helpful discussions. The author is also thankful to the anonymous referees whose suggestions helped to improve the writing of the paper.

\section{REFERENCES}

[1] L. Ambrosio and V. Tortorelli, Approximation of functionals depending on jumps by elliptic functionals via $\Gamma$-convergence. Commun. Pure Appl. Math. 43 (1990) 999-1036.

[2] B. Bourdin, G.A. Francfort and J.-J. Marigo, Numerical experiments in revisited brittle fracture. J. Mech. Phys. Solids 48 (2000) 797-826.

[3] B. Bourdin, C.J. Larsen and C. Richardson, A time-discrete model for dynamic fracture based on crack regularization. Int. J. Fracture 168 (2011) 133-143.

[4] A. Braides, $\Gamma$-convergence for beginners. Vol. 22 of Oxford Lect. Ser. Math. Appl. Oxford University Press, Oxford (2002).

[5] A. Chambolle, A density result in two-dimensional linearized elasticity, and applications. Arch. Rational. Mech. Anal. 167 (2003) 211-233.

[6] G. Dal Maso and R. Toader, A model for the quasi-static growth of brittle fractures based on local minimization. Math. Models Methods Appl. Sci. 12 (2002) 1773-1799.

[7] G. Dal Maso and R. Toader, A model for the quasi-static growth of brittle fractures: existence and approximation results. Arch. Rational. Mech. Anal. 162 (2002) 101-135.

[8] G. Dal Maso, G.A. Francfort and R. Toader, Quasistatic crack growth in nonlinear elasticity. Arch. Rational. Mech. Anal. 176 (2005) 165-225.

[9] E. De Giorgi and G. Dal Maso, $\Gamma$-convergence and calculus of variations. In Mathematical theories of optimization (Genova, 1981). Vol. 979 of Lect. Notes Math. Springer, Berlin (1983) 121-143.

[10] G.A. Francfort and J.-J. Marigo, Revisiting brittle fracture as an energy minimization problem. J. Mech. Phys. Solids 46 (1998) 1319-1342.

[11] G.A. Francfort and C.J. Larsen, Existence and convergence for quasi-static evolution in brittle fracture. Commun. Pure Appl. Math. 56 (2003) 1465-1500.

[12] A. Giacomini, Ambrosio-Tortorelli approximation of quasi-static evolution of brittle fractures. Calc. Var. Partial Differ. Equ. 22 (2005) 129-172.

[13] A. Griffith, The phenomena of rupture and flow in solids. Philos. Trans. Roy. Soc. London (1920) 163-198.

[14] D. Knees, A. Mielke and C. Zanini, On the inviscid limit of a model for crack propagation. Math. Models Methods Appl. Sci. 18 (2008) 1529-1569.

[15] C.J. Larsen, Epsilon-stable quasi-static brittle fracture evolution. Commun. Pure Appl. Math. 63 (2010) 630-654.

[16] C.J. Larsen, C. Ortner and E. Süli, Existence of solutions to a regularized model of dynamic fracture. Math. Models Methods Appl. Sci. 20 (2010) 1021-1048.

[17] A. Mielke, Differential, energetic, and metric formulations for rate-independent processes. In Nonlin. Partial Differ. Equ. Appl. Vol. 2028 of Lect. Notes Math. Springer, Heidelberg (2011) 87-170.

[18] M. Negri and C. Ortner, Quasi-static crack propagation by Griffith's criterion. Math. Models Methods Appl. Sci. 18 (2008) $1895-1925$.

[19] T. Roubíček, Adhesive contact of visco-elastic bodies and defect measures arising by vanishing viscosity. SIAM J. Math. Anal. 45 (2013) 101-126. 